\title{
ANISOTROPIC SOBOLEV CAPACITY WITH FRACTIONAL ORDER
}

\author{
JIE XIAO AND DEPING YE
}

\begin{abstract}
Aвstract. In this paper, we introduce the anisotropic Sobolev capacity with fractional order and develop some basic properties for this new object. Applications to the theory of anisotropic fractional Sobolev spaces are provided. In particular, we give geometric characterizations for a nonnegative Radon measure $\mu$ that naturally induces an embedding of the anisotropic fractional Sobolev class $\dot{\Lambda}_{\alpha, K}^{1,1}$ into the $\mu$-based-Lebesgue-space $L_{\mu}^{n / \beta}$ with $0<\beta \leq n$. Also, we investigate the anisotropic fractional $\alpha$-perimeter. Such a geometric quantity can be used to approximate the anisotropic Sobolev capacity with fractional order. Estimation on the constant in the related Minkowski inequality, which is asymptotically optimal as $\alpha \rightarrow 0^{+}$, will be provided.
\end{abstract}

\section{Anisotropic fractional Sobolev capactity}

A subset $K \subset \mathbb{R}^{n}$ is said to be a convex body if $K$ is a convex compact subset of $\mathbb{R}^{n}$ with nonempty interior. Related to each convex body $K$ with the origin in its interior, one can uniquely define the support function $h_{K}(\cdot): S^{n-1} \rightarrow \mathbb{R}$ as

$$
h_{K}(u)=\max \{\langle y, u\rangle, \quad y \in K\}, \quad \forall u \in S^{n-1},
$$

where $\langle\cdot, \cdot\rangle$ denotes the usual inner product on $\mathbb{R}^{n}$ and induces the usual Euclidean norm $\|\cdot\|$. The unit Euclidean ball of $\mathbb{R}^{n}$ is $B_{2}^{n}=\left\{x \in \mathbb{R}^{n}:\|x\| \leq 1\right\}$. For a subset $L \subset \mathbb{R}^{n}$ with the origin in $L$, its polar $L^{*}$ is defined by $L^{*}=\left\{y \in \mathbb{R}^{n}:\langle x, y\rangle \leq 1, \forall x \in L\right\}$. Note that $L^{*}$ is always convex no matter the convexity of $L$. The volume of $K$ is denoted by $V(K)$, and more general, $V(M)$ denotes the appropriate dimensional Hausdorff content of $M$. For a subset $E \subset \mathbb{R}^{n}, \bar{E}$ denotes the closure of $E$.

The Minkowski functional of $K$ is denoted by $\|\cdot\|_{K}$ and is defined as

$$
\|x\|_{K}=\inf \{\lambda>0: x \in \lambda K\},
$$

where $\lambda K=\{\lambda y: y \in K\}$ for $\lambda \in \mathbb{R}$. In particular, if $K=-K$, then $K$ is said to be originsymmetric. It is easy to check that, for any origin-symmetric convex body $K \subset \mathbb{R}^{n},\|\cdot\|_{K}$ defines a norm on $\mathbb{R}^{n}$. The usual Euclidean norm $\|\cdot\|$ is related to $K=B_{2}^{n}$.

Throughout this paper, $\alpha \in(0,1)$ is a constant and $K \subset \mathbb{R}^{n}$ is always assumed to be an originsymmetric convex body. A function $f$ is said to be of $C_{0}^{\infty}$, denoted by $f \in C_{0}^{\infty}$, if $f$ is smooth and has compact support in $\mathbb{R}^{n}$. Consider the following norm for $f \in C_{0}^{\infty}$

$$
\|f\|_{\Lambda_{\alpha, K}^{1,1}}=\int_{\mathbb{R}^{n}} \int_{\mathbb{R}^{n}} \frac{|f(x)-f(y)|}{\|x-y\|_{K}^{n+\alpha}} d x d y .
$$

2010 Mathematics Subject Classification. 52A38, 53A15, 53A30.

Key words and phrases. Sharpness, isoperimetric inequality, fractional Sobolev capacity, fractional perimeter.

Research of JX is supported in part by: NSERC and URP of MUN, Canada.

Research of DY is supported by NSERC, Canada. 
The completion of all functions $f \in C_{0}^{\infty}$ with the above norm is denoted by $\dot{\Lambda}_{\alpha, K}^{1,1}$. Such a function space will be called the anisotropic fractional Sobolev space with respect to $K$ (or the homogeneous $(\alpha, 1,1, K)$-Besov space). Theorems 1 and 2 in [12] imply that

$$
\lim _{\alpha \rightarrow 0^{+}} \alpha\|f\|_{\dot{\Lambda}_{\alpha, K}^{1,1}}=2 n V(K)\|f\|_{L^{1}} \quad \& \quad \lim _{\alpha \rightarrow 1^{-}}(1-\alpha)\|f\|_{\Lambda_{\alpha, K}^{1,1}}=\int_{\mathbb{R}^{n}}\|\nabla f(x)\|_{Z_{1}^{*} K} d x,
$$

where $Z_{1}^{*} K$ is the polar body of $Z_{1} K$ (the moment body of $K$ ) and the support function of $Z_{1} K$ is determined by

$$
h_{Z_{1} K}(x)=\|x\|_{Z_{1}^{*} K}=\frac{n+1}{2} \int_{K}|\langle x, y\rangle| d y, \quad \forall x \in \mathbb{R}^{n} .
$$

The case $K$ being the unit Euclidean ball $B_{2}^{n}$ has been considered in, e.g., [5, 6, 12, 15, 16].

For any given compact subset $L$ of $\mathbb{R}^{n}$, one can define $\operatorname{cap}\left(L ; \dot{\Lambda}_{\alpha, K}^{1,1}\right)$, the anisotropic fractional Sobolev capacity of $L$ with respect to $K$, by

$$
\operatorname{cap}\left(L ; \dot{\Lambda}_{\alpha, K}^{1,1}\right)=\inf \left\{\|f\|_{\dot{\Lambda}_{\alpha, K}^{1,1}}: f \in C_{0}^{\infty} \& f \geq \mathbf{1}_{L}\right\}
$$

Hereafter, $\mathbf{1}_{E}$ denotes the indicator function of $E \subset \mathbb{R}^{n}$. For any compact $L \subset \mathbb{R}^{n}$, formula (1) implies, (see also [13]),

$$
\lim _{\alpha \rightarrow 0^{+}} \alpha \operatorname{cap}\left(L ; \dot{\Lambda}_{\alpha, K}^{1,1}\right)=2 n V(L) V(K) \quad \& \quad \lim _{\alpha \rightarrow 1^{-}}(1-\alpha) \operatorname{cap}\left(L ; \dot{\Lambda}_{\alpha, K}^{1,1}\right)=\operatorname{cap}\left(L ; \dot{W}_{K}^{1,1}\right),
$$

where

$$
\operatorname{cap}\left(L ; \dot{W}_{K}^{1,1}\right)=\inf \left\{\int_{\mathbb{R}^{n}}\|\nabla f(x)\|_{Z_{1}^{*} K} d x: \quad f \in C_{0}^{\infty} \quad \& \quad f \geq \mathbf{1}_{L}\right\} .
$$

For general subset $E \subset \mathbb{R}^{n}$, the anisotropic fractional Sobolev capacity (or the homogeneous end-point Besov capacity) of $E$ with respect to $K$, denoted by cap $\left(E ; \dot{\Lambda}_{\alpha, K}^{1,1}\right)$, can be defined by

$$
\operatorname{cap}\left(E ; \dot{\Lambda}_{\alpha, K}^{1,1}\right)=\inf _{\text {open } O \supseteq E} \operatorname{cap}\left(O ; \dot{\Lambda}_{\alpha, K}^{1,1}\right)=\inf _{\text {open } O \supseteq E}\left(\sup _{\text {compact } L \subseteq O} \operatorname{cap}\left(L ; \dot{\Lambda}_{\alpha, K}^{1,1}\right)\right) .
$$

Similarly, for general subset $E \subset \mathbb{R}^{n}$,

$$
\operatorname{cap}\left(E ; \dot{W}_{K}^{1,1}\right)=\inf _{\text {open } O \supseteq E} \operatorname{cap}\left(O ; \dot{W}_{K}^{1,1}\right)=\inf _{\text {open } O \supseteq E}\left(\sup _{\text {compact } L \subseteq O} \operatorname{cap}\left(L ; \dot{W}_{K}^{1,1}\right)\right) .
$$

See also [1, 2, 3, 17, 19, 22] for special case $K=B_{2}^{n}$.

As a natural outcome of exploring some essential links between [19, 22] and [12, 13], this paper will focus on the above-newly-introduced anisotropic fractional Sobolev capacity, in particular, its immediate applications to the embedding/trace theory of the anisotropic Sobolev space with fractional order. Section 2 is dedicated to some intrinsic properties of the anisotropic Sobolev capacity with fractional order. Section 3 is for the extrinsic nature of the anisotropic Sobolev capacity with fractional order via the so-called anisotropic fractioal perimeter. Moreover, estimation on the constant in the related Minkowski inequality, which is asymptotically optimal as $\alpha \rightarrow 0^{+}$, will be provided. The anisotropic fractional Sobolev inequalities and their geometric counterparts for anisotropic fractional capacity will be discussed in Section 4. 
2. INTRINSIC PROPERTIES

We begin with exploring some intrinsic properties of the anisotropic Sobolev capacity with fractional order.

Theorem 1. The set-function $E \mapsto \operatorname{cap}\left(E ; \dot{\Lambda}_{\alpha, K}^{1,1}\right)$ is nonnegative and has the following properties. (i) Homogeneity: let $r>0$ be a real constant, then

$$
\operatorname{cap}\left(r E ; \dot{\Lambda}_{\alpha, K}^{1,1}\right)=r^{n-\alpha} \operatorname{cap}\left(E ; \dot{\Lambda}_{\alpha, K}^{1,1}\right), \quad \text { and } \operatorname{cap}\left(E ; \dot{\Lambda}_{\alpha, r K}^{1,1}\right)=r^{n+\alpha} \operatorname{cap}\left(E ; \dot{\Lambda}_{\alpha, K}^{1,1}\right) .
$$

Moreover, for all $r, s>0$,

$$
\operatorname{cap}\left(s E ; \dot{\Lambda}_{\alpha, r K}^{1,1}\right)=s^{n-\alpha} r^{n+\alpha} \operatorname{cap}\left(E ; \dot{\Lambda}_{\alpha, K}^{1,1}\right) .
$$

(ii) Monotonicity: for all subsets $E_{1} \subseteq E_{2} \subseteq \mathbb{R}^{n}$, one has

$$
\operatorname{cap}\left(E_{1} ; \dot{\Lambda}_{\alpha, K}^{1,1}\right) \leq \operatorname{cap}\left(E_{2} ; \dot{\Lambda}_{\alpha, K}^{1,1}\right) .
$$

(iii) Subaddivity: for all compact sets $L_{1}, L_{2} \subseteq \mathbb{R}^{n}$, one has

$$
\operatorname{cap}\left(L_{1} \cup L_{2} ; \dot{\Lambda}_{\alpha, K}^{1,1}\right) \leq \operatorname{cap}\left(L_{1} ; \dot{\Lambda}_{\alpha, K}^{1,1}\right)+\operatorname{cap}\left(L_{2} ; \dot{\Lambda}_{\alpha, K}^{1,1}\right) .
$$

(vi) Upper-semi-continuity: for all decreasing sequence $\left\{L_{j}\right\}_{j=1}^{\infty}$ of compact subsets of $\mathbb{R}^{n}$ with $L_{1} \supseteq L_{2} \supseteq L_{3} \supseteq \cdots$, one has

$$
\lim _{j \rightarrow \infty} \operatorname{cap}\left(L_{j} ; \dot{\Lambda}_{\alpha, K}^{1,1}\right)=\operatorname{cap}\left(\cap_{j=1}^{\infty} L_{j} ; \dot{\Lambda}_{\alpha, K}^{1,1}\right) .
$$

Proof. (i) Let $r>0$. First, the desired equality $\operatorname{cap}\left(E ; \dot{\Lambda}_{\alpha, r K}^{1,1}\right)=r^{n+\alpha} \operatorname{cap}\left(E ; \dot{\Lambda}_{\alpha, K}^{1,1}\right)$ follows immediately from $\|x-y\|_{r K}=r^{-1}\|x-y\|_{K}$ for all $x, y \in \mathbb{R}^{n}$.

To prove $\operatorname{cap}\left(r E ; \dot{\Lambda}_{\alpha, K}^{1,1}\right)=r^{n-\alpha} \operatorname{cap}\left(E ; \dot{\Lambda}_{\alpha, K}^{1,1}\right)$, it is enough to prove the equality for compact sets, due to equation (4). Consider $\|g\|_{\Lambda_{\alpha, K}^{1,1}}$ with $g(x)=f(r x)$ as follows:

$$
\begin{aligned}
\|g\|_{\Lambda_{\alpha, K}^{1,1}} & =\int_{\mathbb{R}^{n}} \int_{\mathbb{R}^{n}} \frac{|g(x)-g(y)|}{\|x-y\|_{K}^{n+\alpha}} d x d y \\
& =\int_{\mathbb{R}^{n}} \int_{\mathbb{R}^{n}} \frac{|f(r x)-f(r y)|}{\|r x-r y\|_{K}^{n+\alpha}} r^{\alpha-n} d(r x) d(r y) \\
& =\int_{\mathbb{R}^{n}} \int_{\mathbb{R}^{n}} \frac{|f(x)-f(y)|}{\|x-y\|_{K}^{n+\alpha}} r^{\alpha-n} d x d y \\
& =r^{\alpha-n}\|f\|_{\Lambda_{\alpha, K}^{1,1}} .
\end{aligned}
$$

Hence, for all compact set $L \subset \mathbb{R}^{n}$, one has

$$
\begin{aligned}
\operatorname{cap}\left(r L, \dot{\Lambda}_{\alpha, K}^{1,1}\right) & =\inf \left\{\|f\|_{\Lambda_{\alpha, K}^{1,1}}: \quad f \in C_{0}^{\infty} \quad \& \quad f \geq \mathbf{1}_{r L}\right\} \\
& =\inf \left\{r^{n-\alpha}\|g\|_{\dot{\Lambda}_{\alpha, K}^{1,1}}: \quad f \in C_{0}^{\infty} \quad \& \quad g \geq \mathbf{1}_{L}\right\} \\
& =r^{n-\alpha} \operatorname{cap}\left(L, \dot{\Lambda}_{\alpha, K}^{1,1}\right) .
\end{aligned}
$$

Finally, for all $r, s>0$, one has

$$
\operatorname{cap}\left(s E ; \dot{\Lambda}_{\alpha, r K}^{1,1}\right)=s^{n-\alpha} \operatorname{cap}\left(E ; \dot{\Lambda}_{\alpha, r K}^{1,1}\right)=s^{n-\alpha} r^{n+\alpha} \operatorname{cap}\left(E ; \dot{\Lambda}_{\alpha, K}^{1,1}\right) .
$$

(ii) It is enough to prove the monotonicity for compact sets, again due to equation (4). For two compact sets $L_{1}$ and $L_{2}$ with $L_{1} \subset L_{2}$, it is easily checked that

$$
\left\{f \in C_{0}^{\infty}: f \geq \mathbf{1}_{L_{1}}\right\} \supset\left\{f \in C_{0}^{\infty}: f \geq \mathbf{1}_{L_{2}}\right\} .
$$


Hence,

$$
\begin{aligned}
\operatorname{cap}\left(L_{1}, \dot{\Lambda}_{\alpha, K}^{1,1}\right) & =\inf \left\{\|f\|_{\dot{\Lambda}_{\alpha, K}^{1,1}}: f \in C_{0}^{\infty} \& f \geq \mathbf{1}_{L_{1}}\right\} \\
& \leq \inf \left\{\|f\|_{\dot{\Lambda}_{\alpha, K}^{1,1}}: f \in C_{0}^{\infty} \& f \geq \mathbf{1}_{L_{2}}\right\} \\
& =\operatorname{cap}\left(L_{2}, \dot{\Lambda}_{\alpha, K}^{1,1}\right) .
\end{aligned}
$$

(iii) Without loss of generality, we may assume $\operatorname{cap}\left(L_{j} ; \dot{\Lambda}_{\alpha, K}^{1,1}\right)<\infty$ with $j=1,2$, as otherwise the consequence holds true trivially. For any $\epsilon>0$, there are $f_{1}, f_{2} \in C_{0}^{\infty}$ such that

$$
f_{j} \geq \mathbf{1}_{L_{j}} \&\left\|f_{j}\right\|_{\dot{\Lambda}_{\alpha, K}^{1,1}}<\operatorname{cap}\left(L_{j} ; \dot{\Lambda}_{\alpha, K}^{1,1}\right)+\epsilon, \quad \forall j=1,2 .
$$

Let $f=\max \left\{f_{1}, f_{2}\right\} \in C_{0}^{\infty}$ and clearly the function $f$ satisfies

$$
f \geq \mathbf{1}_{L_{1} \cup L_{2}} \&|f(x)-f(y)| \leq\left|f_{1}(x)-f_{1}(y)\right|+\left|f_{2}(x)-f_{2}(y)\right|, \quad \forall x, y \in \mathbb{R}^{n} .
$$

This further implies

$$
\operatorname{cap}\left(L_{1} \cup L_{2} ; \dot{\Lambda}_{\alpha, K}^{1,1}\right) \leq\|f\|_{\dot{\Lambda}_{\alpha, K}^{1,1}} \leq\left\|f_{1}\right\|_{\dot{\Lambda}_{\alpha, K}^{1,1}}+\left\|f_{2}\right\|_{\dot{\Lambda}_{\alpha, K}^{1,1}} \leq \operatorname{cap}\left(L_{1} ; \dot{\Lambda}_{\alpha, K}^{1,1}\right)+\operatorname{cap}\left(L_{2} ; \dot{\Lambda}_{\alpha, K}^{1,1}\right)+2 \epsilon .
$$

The desired consequence follows by letting $\epsilon \rightarrow 0$.

(iv) Suppose that $\left\{L_{j}\right\}_{j=1}^{\infty}$ is a decreasing sequence of compact subsets of $\mathbb{R}^{n}$. Then, $L=\cap_{j=1}^{\infty} L_{j}$ is compact. For any $\epsilon \in(0,1)$, there is a function $f \in C_{0}^{\infty}$ such that

$$
f \geq \mathbf{1}_{L} \quad \& \quad\|f\|_{\dot{\Lambda}_{\alpha, K}^{1,1}}<\operatorname{cap}\left(L ; \dot{\Lambda}_{\alpha, K}^{1,1}\right)+\epsilon .
$$

Let $L_{f, \epsilon}=:\left\{x \in \mathbb{R}^{n}: f(x) \geq 1-\epsilon\right\}$, which is compact. Due to $L_{j}$ decreasing to $L$, one can find an integer $j>0$ large enough, such that, $L_{j} \subset L_{f, \epsilon}$. By Part (ii) and formula (2), one has,

$$
\lim _{j \rightarrow \infty} \operatorname{cap}\left(L_{j} ; \dot{\Lambda}_{\alpha, K}^{1,1}\right) \leq \operatorname{cap}\left(L_{f, \epsilon} ; \dot{\Lambda}_{\alpha, K}^{1,1}\right) \leq(1-\epsilon)^{-1}\|f\|_{\Lambda_{\alpha, K}^{1,1}} \leq \frac{\operatorname{cap}\left(L ; \dot{\Lambda}_{\alpha, K}^{1,1}\right)+\epsilon}{1-\epsilon} .
$$

Letting $\epsilon \rightarrow 0$ and again by Part (ii), we get

$$
\operatorname{cap}\left(L ; \dot{\Lambda}_{\alpha, K}^{1,1}\right) \leq \lim _{j \rightarrow \infty} \operatorname{cap}\left(L_{j} ; \dot{\Lambda}_{\alpha, K}^{1,1}\right) \leq \operatorname{cap}\left(L ; \dot{\Lambda}_{\alpha, K}^{1,1}\right),
$$

and hence equality holds.

Remark 1. Along similar lines, one can prove analogous intrinsic result for the anisotropic Sobolev capacity cap $\left(\cdot ; \dot{W}_{K}^{1,1}\right)$, with $\dot{\Lambda}_{\alpha, K}^{1,1}$ and $n \pm \alpha$ in Theorem 1 replaced by $\dot{W}_{K}^{1,1}$ and $n \pm 1$ respectively.

\section{EXTRINSIC PROPERTIES}

In this section, we will reveal an extrinsic nature of the anisotropic Sobolev capacity with fractional order via the so-called anisotropic fractional perimeter.

For a set $E \subseteq \mathbb{R}^{n}$, let $E^{c}=\mathbb{R}^{n} \backslash E$ be the complement of $E \subset \mathbb{R}^{n}$. Define $P_{\alpha}(E, K)$, the anisotropic fractional $\alpha$-perimeter of $E$ with respect to $K$ [13], as

$$
P_{\alpha}(E, K)=\int_{E} \int_{E^{c}} \frac{1}{\|x-y\|_{K}^{n+\alpha}} d x d y=\frac{\left\|\mathbf{1}_{E}\right\|_{\Lambda_{\alpha, K}^{1,1}}}{2} .
$$

Theorems 4 and 6 in [13] assert that, if $E \subset \mathbb{R}^{n}$ is a bounded Borel set of finite perimeter, then

$$
\lim _{\alpha \rightarrow 0^{+}} \alpha P_{\alpha}(E, K)=n V(E) V(K) \quad \& \quad \lim _{\alpha \rightarrow 1^{-}}(1-\alpha) P_{\alpha}(E, K)=P\left(E, Z_{1} K\right) .
$$


Here and henceforth, $P(E, F)$ stands for the anisotropic perimeter of a Borel set $E \subset \mathbb{R}^{n}$ with respect to an origin-symmetric convex body $F$, which has the following form:

$$
P(E, F)=\int_{\partial^{*} E}\left\|v_{E}(x)\right\|_{F^{*}} d \mathcal{H}^{n-1}(x),
$$

with $\mathcal{H}^{n-1}$ the $(n-1)$ dimensional Hausdorff measure, $v_{E}(x)$ the measure theoretic outer unit normal of $E$ at point $x$ in $\partial^{*} E$, the reduced boundary of $E$. In particular, $P(E)=P\left(E, B_{2}^{n}\right)$ is called the perimeter of $E$. When $\partial E$, the boundary of $E$, is smooth, $P(E)$ is equal to the usual surface area of $\partial E$. On the other hand, $P(E, F)$ equals the classical mixed volume of $E$ and $F$, if $E$ is also a convex body. The special case $P_{\alpha}(E)=P_{\alpha}\left(E, B_{2}^{n}\right)$, named as the fractional $\alpha$-perimeter of $E$ (cf. [10]), is a classical object and receives a lot of attention. In particular, by formula (5), one has,

$$
\lim _{\alpha \rightarrow 0^{+}} \alpha P_{\alpha}(E)=n V\left(B_{2}^{n}\right) V(E) \quad \& \quad \lim _{\alpha \rightarrow 1^{-}}(1-\alpha) P_{\alpha}(E)=2^{-1} \tau_{n} P(E),
$$

where $\tau_{n}=\int_{\mathbb{S}^{n-1}}|\cos (\theta)| d \sigma$ with $\theta$ being the angle deviation from the vertical direction and $d \sigma$ being the standard area measure on the unit sphere $\mathbb{S}^{n-1}$ of $\mathbb{R}^{n}$; see [15, 16].

The following cyclic inequality for the anisotropic fractional perimeters holds.

Proposition 2. Let $0<\alpha<\beta<\gamma<1$. For all $E \subset \mathbb{R}^{n}$, one has,

$$
\left[P_{\beta}(E, K)\right]^{\gamma-\alpha} \leq\left[P_{\alpha}(E, K)\right]^{\gamma-\beta}\left[P_{\gamma}(E, K)\right]^{\beta-\alpha} .
$$

Proof. Let $0<\alpha<\beta<\gamma<1$ which implies $0<\frac{\beta-\alpha}{\gamma-\alpha}<1$. By Hölder's inequality, one has,

$$
\begin{aligned}
P_{\beta}(E, K) & =\int_{E} \int_{E^{c}} \frac{1}{\|x-y\|_{K}^{n+\beta}} d x d y \\
& =\int_{E} \int_{E^{c}}\left(\frac{1}{\|x-y\|_{K}^{n+\alpha}}\right)^{\frac{\gamma-\beta}{\gamma-\alpha}}\left(\frac{1}{\|x-y\|_{K}^{n+\gamma}}\right)^{\frac{\beta-\alpha}{\gamma-\alpha}} d x d y \\
& \leq\left(\int_{E} \int_{E^{c}} \frac{1}{\|x-y\|_{K}^{n+\alpha}} d x d y\right)^{\frac{\gamma-\beta}{\gamma-\alpha}}\left(\int_{E} \int_{E^{c}} \frac{1}{\|x-y\|_{K}^{n+\gamma}} d x d y\right)^{\frac{\beta-\alpha}{\gamma-\alpha}} \\
& =\left(P_{\alpha}(E, K)\right)^{\frac{\gamma-\beta}{\gamma-\alpha}}\left(P_{\gamma}(E, K)\right)^{\frac{\beta-\alpha}{\gamma-\alpha}} .
\end{aligned}
$$

The desired inequality follows by taking power $\gamma-\alpha$ from both sides.

For bounded open set $E \subset \mathbb{R}^{n}$ with $V(\partial E)=V(\bar{E} \backslash E)=0$, one has

$$
P_{\alpha}(\bar{E}, K)=P_{\alpha}(E, K) .
$$

In fact, for all (fixed) $y \in E \cup \bar{E}^{c}$, there is $r>0$, such that $\|y-x\|_{K}>r$ for all $x \in \bar{E} \backslash E$ as $E \cup \bar{E}^{c}$ is open. Hence, for all $y \in E \cup \bar{E}^{c}$,

$$
0 \leq \int_{\bar{E} \backslash E} \frac{1}{\|x-y\|_{K}^{n+\alpha}} d x \leq \int_{\bar{E} \backslash E} \frac{1}{r^{n+\alpha}} d x=\frac{V(\bar{E} \backslash E)}{r^{n+\alpha}}=0 .
$$

This further implies that

$$
\int_{\bar{E}^{c}}\left(\int_{\bar{E} \backslash E} \frac{1}{\|x-y\|_{K}^{n+\alpha}} d x\right) d y=\int_{E}\left(\int_{\bar{E} \backslash E} \frac{1}{\|x-y\|_{K}^{n+\alpha}} d x\right) d y=0,
$$


and thus, the desired formula (6) holds:

$$
\begin{aligned}
P_{\alpha}(\bar{E}, K)-P_{\alpha}(E, K) & =\int_{\bar{E}} \int_{\bar{E}^{c}} \frac{1}{\|x-y\|_{K}^{n+\alpha}} d x d y-\int_{E} \int_{E^{c}} \frac{1}{\|x-y\|_{K}^{n+\alpha}} d x d y \\
& =\int_{\bar{E}^{c}}\left(\int_{\bar{E} \backslash E} \frac{1}{\|x-y\|_{K}^{n+\alpha}} d x\right) d y-\int_{E}\left(\int_{\bar{E} \backslash E} \frac{1}{\|x-y\|_{K}^{n+\alpha}} d x\right) d y \\
& =0 .
\end{aligned}
$$

Similar to the proof of Theorem 1, $P_{\alpha}(E, K)$ has the following homogeneity: for all $r, s>0$,

$$
P_{\alpha}(s E, r K)=s^{n-\alpha} r^{n+\alpha} P_{\alpha}(E, K) .
$$

It is known that $P_{\alpha}(E, K) \geq \gamma_{\alpha}(K) V(E)^{\frac{n-\alpha}{n}}$ holds true for every bound Borel set $E \subset \mathbb{R}^{n}$ with $\gamma_{\alpha}(K)>0$ a constant defined by (cf. [13])

$$
\gamma_{\alpha}(K)=\inf \left\{P_{\alpha}(E, K) V(E)^{-\frac{n-\alpha}{n}}: E \subset \Omega, V(E)>0\right\},
$$

where $\Omega$ is a given and fixed open bounded subset of $\mathbb{R}^{n}$. As claimed in [13], the constant $\gamma_{\alpha}(K)$ defined in formula (8) only depends on $K$ and is independent of the choice of $\Omega$. Heuristically, formula (7) indicates that $\gamma_{\alpha}(K) V(K)^{-\frac{n+\alpha}{n}}$ may be even independent of $K$.

Following the idea of verifying [7, Lemma 6.1], we establish the following anisotropic isoperimetric inequality for $P_{\alpha}(E, K)$, which provides an estimate for the constant $\gamma_{\alpha}(K)$.

Theorem 3. Let $E$ be a bounded Borel subset of $\mathbb{R}^{n}$. The following anisotropic isoperimetric inequality with fractional order $\alpha \in(0,1)$ holds:

$$
\alpha P_{\alpha}(E, K) \geq n V(K)^{\frac{n+\alpha}{n}} V(E)^{\frac{n-\alpha}{n}} .
$$

Moreover, this inequality is asymptotically optimal in the sense of

$$
\lim _{\alpha \rightarrow 0^{+}} \alpha P_{\alpha}(E, K)=\lim _{\alpha \rightarrow 0^{+}} n V(K)^{\frac{n+\alpha}{n}} V(E)^{\frac{n-\alpha}{n}}=n V(K) V(E) .
$$

Proof. Let $E$ be a bounded Borel subset of $\mathbb{R}^{n}$. The desired inequality holds trivially if $V(E)=0$. Now let us consider $0<V(E)<\infty$, and let $r=\left(\frac{V(E)}{V(K)}\right)^{1 / n}>0$. For any fixed $x \in E$, let

$$
B_{r}(x)=\left\{z \in \mathbb{R}^{n}:\|z-x\|_{K} \leq r\right\} .
$$

In fact, the volume of $K$ is equal to $V\left(\left\{z:\|z\|_{K} \leq 1\right\}\right)$ and hence the volume of $B_{r}(x)$ equals $V(E)$. This further implies

$$
\begin{aligned}
V\left(E^{c} \cap B_{r}(x)\right) & =V\left(B_{r}(x) \backslash E\right) \\
& =V\left(B_{r}(x)\right)-V\left(E \cap B_{r}(x)\right) \\
& =V(E)-V\left(E \cap B_{r}(x)\right) \\
& =V\left(E \backslash B_{r}(x)\right) \\
& =V\left(B_{r}(x)^{c} \cap E\right) .
\end{aligned}
$$

Note that $\|y-x\|_{K} \leq r$ for $y \in E^{c} \cap B_{r}(x)$ and $\|y-x\|_{K}>r$ for $y \in B_{r}(x)^{c} \cap E$. Thus,

$$
\begin{aligned}
\int_{E^{c} \cap B_{r}(x)} \frac{d y}{\|x-y\|_{K}^{n+\alpha}} & \geq \int_{E^{c} \cap B_{r}(x)} \frac{d y}{r^{n+\alpha}} \\
& =\frac{V\left(E^{c} \cap B_{r}(x)\right)}{r^{n+\alpha}} \\
& =\frac{V\left(B_{r}(x)^{c} \cap E\right)}{r^{n+\alpha}}
\end{aligned}
$$




$$
\begin{aligned}
& =\int_{B_{r}(x)^{c} \cap E} \frac{d y}{r^{n+\alpha}} \\
& \geq \int_{B_{r}(x)^{c} \cap E} \frac{d y}{\|x-y\|_{K}^{n+\alpha}} .
\end{aligned}
$$

This in turn implies

$$
\begin{aligned}
\int_{E^{c}} \frac{d y}{\|x-y\|_{K}^{n+\alpha}} & =\int_{E^{c} \cap B_{r}(x)} \frac{d y}{\|x-y\|_{K}^{n+\alpha}}+\int_{E^{c} \cap B_{r}(x)^{c}} \frac{d y}{\|x-y\|_{K}^{n+\alpha}} \\
& \geq \int_{B_{r}(x)^{c} \cap E} \frac{d y}{\|x-y\|_{K}^{n+\alpha}}+\int_{E^{c} \cap B_{r}(x)^{c}} \frac{d y}{\|x-y\|_{K}^{n+\alpha}} \\
& =\int_{B_{r}(x)^{c}} \frac{d y}{\|x-y\|_{K}^{n+\alpha}},
\end{aligned}
$$

where the last integral can be calculated by Fubini's theorem as follows:

$$
\begin{aligned}
\int_{B_{r}(x)^{c}} \frac{d y}{\|x-y\|_{K}^{n+\alpha}} & =\int_{\left\{y:\|y-x\|_{K}>r\right\}} \frac{d y}{\|x-y\|_{K}^{n+\alpha}} \\
& =\int_{\left\{y:\|y-x\|_{K}>r\right\}}\left(\int_{\|y-x\|_{K}}^{\infty}(n+\alpha) t^{-n-\alpha-1} d t\right) d y \\
& =\int_{r}^{\infty}(n+\alpha) t^{-n-\alpha-1}\left(\int_{\left\{y: r<\|y-x\|_{K} \leq t\right\}} d y\right) d t \\
& =V(K) \int_{r}^{\infty}(n+\alpha) t^{-n-\alpha-1}\left(t^{n}-r^{n}\right) d t \\
& =\frac{n}{\alpha} \cdot r^{-\alpha} V(K) \\
& =\frac{n}{\alpha} \cdot \frac{V(K)^{1+\alpha / n}}{V(E)^{\alpha / n}} .
\end{aligned}
$$

Hence, one gets

$$
P_{\alpha}(E, K)=\int_{E}\left(\int_{E^{c}} \frac{d y}{\|x-y\|_{K}^{n+\alpha}}\right) d x \geq \int_{E}\left(\int_{B_{r}(x)^{c}} \frac{d y}{\|x-y\|_{K}^{n+\alpha}}\right) d x \geq \frac{n}{\alpha} \cdot V(K)^{\frac{n+\alpha}{n}} V(E)^{\frac{n-\alpha}{n}} .
$$

The asymptotic optimality is a direct consequence of formula (5), i.e.,

$$
n V(E) V(K)=\lim _{\alpha \rightarrow 0^{+}} \alpha P_{\alpha}(E, K) \geq \lim _{\alpha \rightarrow 0^{+}} n V(K)^{\frac{n+\alpha}{n}} V(E)^{\frac{n+\alpha}{n}}=n V(E) V(K) .
$$

The definition for $\gamma_{\alpha}(K)$ and Theorem 3 imply that

$$
\frac{n}{\alpha} V(K)^{\frac{n+\alpha}{n}} \leq \inf \left\{P_{\alpha}(E, K) V(E)^{-\frac{n-\alpha}{n}}: E \subset \Omega, V(E)>0\right\}=\gamma_{\alpha}(K) .
$$

That is, we have a lower bound for $\gamma_{\alpha}(K)$ :

$$
\gamma_{\alpha}(K) \geq \frac{n}{\alpha} V(K)^{\frac{n+\alpha}{n}}
$$

Remark 2. It is well known that the anisotropic isoperimetric inequality (cf. [9, (1.4)])

$$
P(E, K) \geq n V(K)^{\frac{1}{n}} V(E)^{\frac{n-1}{n}}
$$


can be obtained by the classical Brunn-Minkowski inequality [8]. However, such an inequality cannot be obtained from Theorem 3 by letting $\alpha \rightarrow 1^{-}$, if one notices the second limit of (5). On the other hand, inequalities in Theorem 3 and the anisotropic isoperimetric inequality have two common features: the dimension $n$ appears in front of the products of the powered volumes, and the sums of the powers of $V(K)$ and $V(E)$ are constants:

$$
\frac{n+\alpha}{n}+\frac{n-\alpha}{n}=2 \quad \& \quad \frac{1}{n}+\frac{n-1}{n}=1 .
$$

As in [9], it may be interesting to study the deficit:

$$
\frac{\alpha P_{\alpha}(E, K)}{n V(K)^{\frac{n+\alpha}{n}} V(E)^{\frac{n-\alpha}{n}}}-1 ;
$$

see [10] for a PDE-based treatment of such a question with $K=B_{2}^{n}$. We leave this for future investigation.

The relation between the anisotropic fractional Sobolev capacity and the anisotropic factional perimeter is stated in the following theorem, which is an extension of [22, Theorem 2] for $K=B_{2}^{n}$.

Theorem 4. Let L be a compact subset of $\mathbb{R}^{n}$. Then

$$
\operatorname{cap}\left(L ; \dot{\Lambda}_{\alpha, K}^{1,1}\right)=2 \inf _{O \in \mathrm{O}^{\infty}(L)} P_{\alpha}(O, K),
$$

where $\mathrm{O}^{\infty}(L)$ denotes the class of all open sets with $C^{\infty}$ boundary that contain $L$.

Proof. Let $L \subset \mathbb{R}^{n}$ be compact. For $f \in C_{0}^{\infty}$ with $f \geq \mathbf{1}_{L}$, one has

$$
L \subset\left\{x \in \mathbb{R}^{n}: f(x)>t\right\}, \quad \forall t \in(0,1) .
$$

The generalized co-area formula in [18] (see also [13]) implies

$$
\begin{aligned}
\|f\|_{\dot{\Lambda}_{\alpha, K}^{1,1}} & =2 \int_{0}^{\infty} P_{\alpha}\left(\left\{x \in \mathbb{R}^{n}: f(x)>t\right\}, K\right) d t \\
& \geq 2 \int_{0}^{1} P_{\alpha}\left(\left\{x \in \mathbb{R}^{n}: f(x)>t\right\}, K\right) d t \\
& \geq 2 \inf _{O \in \mathrm{O}^{\infty}(L)} P_{\alpha}(O, K),
\end{aligned}
$$

where the last inequality follows from

$$
\left\{x \in \mathbb{R}^{n}: f(x)>t\right\} \in \mathrm{O}^{\infty}(L) .
$$

Hence, formula (2) implies

$$
\operatorname{cap}\left(L ; \dot{\Lambda}_{\alpha, K}^{1,1}\right) \geq 2 \inf _{O \in \mathrm{O}^{\infty}(L)} P_{\alpha}(O, K) .
$$

On the other hand, similar to the proof of Theorem 3.1 in [11] (or the proof of Part (ii) of Theorem 7 in this paper), one can prove that

$$
\operatorname{cap}\left(L ; \dot{\Lambda}_{\alpha, K}^{1,1}\right) \leq \operatorname{cap}\left(\bar{O} ; \dot{\Lambda}_{\alpha, K}^{1,1}\right) \leq 2 P_{\alpha}(O, K), \quad \forall O \in \mathrm{O}^{\infty}(L),
$$

where the first inequality is by Part (ii) of Theorem 1 . This further implies that

$$
\operatorname{cap}\left(L ; \dot{\Lambda}_{\alpha, K}^{1,1}\right) \leq 2 \inf _{O \in \mathrm{O}^{\infty}(L)} P_{\alpha}(O, K),
$$

and the desired formula for $\operatorname{cap}\left(L ; \dot{\Lambda}_{\alpha, K}^{1,1}\right)$ follows. 
Remark 3. Combining formula (1) and the first limit of [13, p.90, line 5], we can prove the following co-area formula

$$
\int_{\mathbb{R}^{n}}\|\nabla f(x)\|_{Z_{1}^{*} K} d x=2 \int_{0}^{\infty} P\left(\left\{x \in \mathbb{R}^{n}: f(x)>t\right\}, Z_{1} K\right) d t .
$$

Moreover, Theorem 4 together with formulas (1), (3) and (5) imply that

$$
\operatorname{cap}\left(L ; \dot{W}_{K}^{1,1}\right)=2 \inf _{O \in \mathrm{O}^{\infty}(L)} P\left(O, Z_{1} K\right),
$$

which extends [14, Lemma 2.2.5] for $K=B_{2}^{n}$ to the anisotropic case.

We now establish the anisotropic isocapacitary inequality with fractional order $\alpha \in(0,1)$.

Corollary 5. Let L be a compact subset of $\mathbb{R}^{n}$. Then, the following anisotropic isocapacitary inequality with fractional order $\alpha \in(0,1)$ holds:

$$
\alpha \operatorname{cap}\left(L ; \dot{\Lambda}_{\alpha, K}^{1,1}\right) \geq 2 n V(K)^{\frac{n+\alpha}{n}} V(L)^{\frac{n-\alpha}{n}} .
$$

Moreover, this inequality is asymptotically optimal in the sense of:

$$
\lim _{\alpha \rightarrow 0^{+}} \alpha \operatorname{cap}\left(L ; \dot{\Lambda}_{\alpha, K}^{1,1}\right)=\lim _{\alpha \rightarrow 0^{+}} 2 n V(K)^{\frac{n+\alpha}{n}} V(L)^{\frac{n-\alpha}{n}}=2 n V(K) V(L) .
$$

Proof. Combining Theorems 3 and 4 , one has

$$
\begin{aligned}
\operatorname{cap}\left(L ; \dot{\Lambda}_{\alpha, K}^{1,1}\right) & =2 \inf _{O \in \mathrm{O}^{\infty}(L)} P_{\alpha}(O, K) \\
& \geq \inf _{O \in \mathrm{O}^{\infty}(L)}\left(2 \gamma_{\alpha}(K) V(O)^{\frac{n-\alpha}{n}}\right) \\
& \geq 2 \gamma_{\alpha}(K) V(L)^{\frac{n-\alpha}{n}} \\
& \geq \frac{2 n}{\alpha} \cdot V(K)^{\frac{n+\alpha}{n}} V(L)^{\frac{n-\alpha}{n}} .
\end{aligned}
$$

Together with formula (3), one has

$$
2 n V(L) V(K)=\lim _{\alpha \rightarrow 0^{+}} \alpha \operatorname{cap}\left(L ; \dot{\Lambda}_{\alpha, K}^{1,1}\right) \geq \lim _{\alpha \rightarrow 0^{+}} 2 n V(K)^{\frac{n+\alpha}{n}} V(L)^{\frac{n-\alpha}{n}}=2 n V(L) V(K) .
$$

Remark 4. Similarly, inequality (9) and formula (11) imply the following anisotropic isocapacitary inequality:

$$
\operatorname{cap}\left(L ; \dot{W}_{K}^{1,1}\right) \geq 2 n V\left(Z_{1} K\right)^{\frac{1}{n}} V(L)^{\frac{n-1}{n}} .
$$

\section{Anisotropic Fractional Sobolev EMBEDdings}

This section dedicates to establish the anisotropic fractional Sobolev inequalities (generated by the Radon-measure-based-Lebesgue-space $L_{\mu}^{n / \beta}$ on $\mathbb{R}^{n}$ ) and their geometric counterparts for anisotropic fractional capacity.

First, we have the anisotropic extension of [22, Theorem 3(i)].

Theorem 6. Let $\mu$ be a nonnegative Radon measure on $\mathbb{R}^{n}$, and let $0<\beta<\infty$ and $\kappa_{n, \alpha, \beta}>0$ be constants. Then the following two inequalities are equivalent:

(i) The analytic inequality

$$
\|f\|_{L_{\mu}^{\frac{n}{\beta}}} \leq \kappa_{n, \alpha, \beta}\left(\int_{0}^{\infty}\left(\operatorname{cap}\left(\left\{x \in \mathbb{R}^{n}:|f(x)| \geq t\right\} ; \dot{\Lambda}_{\alpha, K}^{1,1}\right)\right)^{\frac{n}{\beta}} d t^{\frac{n}{\beta}}\right)^{\frac{\beta}{n}}, \forall f \in C_{0}^{\infty} ;
$$


(ii) The geometric inequality

(13) $\quad(\mu(\bar{O}))^{\frac{\beta}{n}} \leq \kappa_{n, \alpha, \beta} \operatorname{cap}\left(\bar{O} ; \dot{\Lambda}_{\alpha, K}^{1,1}\right)$, for all bounded domain $O \subset \mathbb{R}^{n}$ with $C^{\infty}$ boundary $\partial O$.

Proof. By Fubini's theorem, one has, for all $f \in C_{0}^{\infty}$,

$$
\begin{aligned}
\|f\|_{L_{\mu}^{\frac{n}{\beta}}} & =\left(\int_{\mathbb{R}^{n}}|f(x)|^{\frac{n}{\beta}} d \mu(x)\right)^{\frac{\beta}{n}} \\
& =\left(\int_{\mathbb{R}^{n}}\left[\int_{0}^{|f(x)|} n \beta^{-1} t^{\frac{n}{\beta}-1} d t\right] d \mu(x)\right)^{\frac{\beta}{n}} \\
& =\left(\int_{0}^{\infty}\left[\int_{O_{t}(f)} n \beta^{-1} t^{\frac{n}{\beta}-1} d \mu(x)\right] d t\right)^{\frac{\beta}{n}} \\
& =\left(\int_{0}^{\infty} \mu\left(O_{t}(f)\right) d t^{\frac{n}{\beta}}\right)^{\frac{\beta}{n}},
\end{aligned}
$$

where, for all $t>0, O_{t}(f)$ and $d t^{\frac{n}{\beta}}$ are defined as

$$
O_{t}(f)=\left\{x \in \mathbb{R}^{n}:|f(x)|>t\right\} \quad \& \quad d t^{\frac{n}{\beta}}=n \beta^{-1} t^{\frac{n}{\beta}-1} d t .
$$

(ii) $\Rightarrow$ (i) Suppose that inequality (13) holds. Note that, for $f \in C_{0}^{\infty}$, the set $O_{t}(f)$ is a bounded open domain with $C^{\infty}$ boundary. Together with inequality (13) and formula (14), one gets the desired inequality (12) as follows:

$$
\begin{aligned}
\|f\|_{L_{\mu}^{\frac{n}{\beta}}} & =\left(\int_{0}^{\infty} \mu\left(O_{t}(f)\right) d t^{\frac{n}{\beta}}\right)^{\frac{\beta}{n}} \\
& \leq\left(\int_{0}^{\infty} \mu\left(\overline{O_{t}(f)}\right) d t^{\frac{n}{\beta}}\right)^{\frac{\beta}{n}} \\
& \leq \kappa_{n, \alpha, \beta}\left(\int_{0}^{\infty}\left(\operatorname{cap}\left(\overline{O_{t}(f)} ; \dot{\Lambda}_{\alpha, K}^{1,1}\right)\right)^{\frac{n}{\beta}} d t^{\frac{n}{\beta}}\right)^{\frac{\beta}{n}} .
\end{aligned}
$$

(i) $\Rightarrow$ (ii) Suppose that inequality (12) holds. For any bounded domain $O \subset \mathbb{R}^{n}$ with $C^{\infty}$ boundary $\partial O$ and $0<\epsilon<1$, let

$$
f_{\epsilon}(x)= \begin{cases}1-\epsilon^{-1} \operatorname{dist}(x, \bar{O}), & \text { if } \operatorname{dist}(x, \bar{O})<\epsilon \\ 0, & \text { if } \operatorname{dist}(x, \bar{O}) \geq \epsilon\end{cases}
$$

where $\operatorname{dist}(x, E)$ denotes the Euclidean distance of a point $x$ to a set $E$. One can check that $f_{\epsilon} \in C_{0}^{\infty}$ and hence inequality (12) holds for $f_{\epsilon}$. Moreover,

$$
(\mu(\bar{O}))^{\frac{\beta}{n}}=\lim _{\epsilon \rightarrow 0^{+}}\left\|f_{\epsilon}\right\|_{L_{\mu}^{\frac{n}{\beta}}} .
$$

Let $O_{\epsilon}=\left\{x \in \mathbb{R}^{n}: \operatorname{dist}(x, \bar{O})<\epsilon\right\}$. Inequality (12) implies that for all $0<\epsilon<1$,

$$
\begin{aligned}
\left\|f_{\epsilon}\right\|_{L_{\mu}^{\frac{n}{\beta}}} & \leq \kappa_{n, \alpha, \beta}\left(\int_{0}^{\infty}\left(\operatorname{cap}\left(\overline{O_{t}\left(f_{\epsilon}\right)} ; \dot{\Lambda}_{\alpha, K}^{1,1}\right)\right)^{\frac{n}{\beta}} d t^{\frac{n}{\beta}}\right)^{\frac{\beta}{n}} \\
& =\kappa_{n, \alpha, \beta}\left(\int_{0}^{1}\left(\operatorname{cap}\left(\overline{O_{t}\left(f_{\epsilon}\right)} ; \dot{\Lambda}_{\alpha, K}^{1,1}\right)\right)^{\frac{n}{\beta}} d t^{\frac{n}{\beta}}\right)^{\frac{\beta}{n}}
\end{aligned}
$$




$$
\leq \kappa_{n, \alpha, \beta} \operatorname{cap}\left(\overline{O_{\epsilon}} ; \dot{\Lambda}_{\alpha, K}^{1,1}\right),
$$

where the last inequality is due to Part (ii) of Theorem 1 and $\overline{O_{t}\left(f_{\epsilon}\right)} \subset \overline{O_{\epsilon}}$. Taking $\epsilon \rightarrow 0^{+}$, one gets inequality (13) by Part (iv) of Theorem 1 and formula (15).

As a matter of fact, both inequalities (12) and (13) hold true for $\mu$ being the Lebesgue measure on $\mathbb{R}^{n}$ with constant $\kappa_{n, \alpha, n-\alpha}=\left(2 \gamma_{\alpha}(K)\right)^{-1}$. Moreover, if the nonnegative Radon measure $\mu$ is absolutely continuous with respect to the Lebesgue measure on $\mathbb{R}^{n}$ and $f(x)=\frac{d \mu}{d x}$ is bounded on $\mathbb{R}^{n}$, then inequalities (12) and (13) hold true for some constant $\kappa_{n, \alpha, n-\alpha}$. To this end, it can be seen from the proof of Corollary 5 that for all bounded domain $O \subset \mathbb{R}^{n}$ with $C^{\infty}$ boundary $\partial O$,

$$
(V(O))^{\frac{n-\alpha}{n}}=(V(\bar{O}))^{\frac{n-\alpha}{n}} \leq\left(2 \gamma_{\alpha}(K)\right)^{-1} \operatorname{cap}\left(\bar{O} ; \dot{\Lambda}_{\alpha, K}^{1,1}\right) .
$$

That is, inequality (13) holds true with constant $\kappa_{n, \alpha, n-\alpha}=\left(2 \gamma_{\alpha}(K)\right)^{-1}$, and so does inequality (12) by Theorem 6 . Moreover, let $\mu$ be such that $f(x)=\frac{d \mu}{d x}$ is bounded on $\mathbb{R}^{n}$, say by $M<\infty$. For all bounded domain $O \subset \mathbb{R}^{n}$ with $C^{\infty}$ boundary $\partial O$, one has, $\mu(\bar{O}) \leq M V(\bar{O})$, and hence,

$$
(\mu(\bar{O}))^{\frac{n-\alpha}{n}} \leq M^{\frac{n-\alpha}{n}}(V(\bar{O}))^{\frac{n-\alpha}{n}} \leq M^{\frac{n-\alpha}{n}}\left(2 \gamma_{\alpha}(K)\right)^{-1} \operatorname{cap}\left(\bar{O} ; \dot{\Lambda}_{\alpha, K}^{1,1}\right) .
$$

That is, inequality (13) holds true for $\mu$ with constant $\kappa_{n, \alpha, n-\alpha}=M^{\frac{n-\alpha}{n}}\left(2 \gamma_{\alpha}(K)\right)^{-1}$, and so does inequality (12) by Theorem 6 .

Remark 5. Similar to Theorem 6 and comments after, one can get analogous results for the anisotropic fractional Sobolev capacity $\operatorname{cap}\left(\cdot, \dot{W}_{K}^{1,1}\right)$. More precisely, with $\mu$ and $\beta$ as in Theorem 6 and $\kappa_{n, \beta}$ a constant, the following two inequalities are equivalent:

(i) For all $f \in C_{0}^{\infty}$,

$$
\|f\|_{L_{\mu}^{\frac{n}{\beta}}} \leq \kappa_{n, \beta}\left(\int_{0}^{\infty}\left(\operatorname{cap}\left(\left\{x \in \mathbb{R}^{n}:|f(x)| \geq t\right\} ; \dot{W}_{K}^{1,1}\right)\right)^{\frac{n}{\beta}} d t^{\frac{n}{\beta}}\right)^{\frac{\beta}{n}} ;
$$

(ii) For all bounded domain $O \subset \mathbb{R}^{n}$ with $C^{\infty}$ boundary $\partial O$,

$$
(\mu(\bar{O}))^{\frac{\beta}{n}} \leq \kappa_{n, \beta} \operatorname{cap}\left(\bar{O} ; \dot{W}_{K}^{1,1}\right) .
$$

Moreover, the above inequalities hold for $\mu$ being the Lebesgue measure on $\mathbb{R}^{n}$ with constant $\kappa_{n, n-1}=\left(2 n V\left(Z_{1} K\right)^{\frac{1}{n}}\right)^{-1}$.

Second, we have the anisotropic version of [22, Theorem 3 (ii)].

Theorem 7. Let $0<\beta<\infty$. The following inequalities hold and are equivalent:

(i) The analytic inequality

$$
\left(\int_{0}^{\infty}\left(\operatorname{cap}\left(\left\{x \in \mathbb{R}^{n}:|f(x)| \geq t\right\} ; \dot{\Lambda}_{\alpha, K}^{1,1}\right)\right)^{\frac{n}{\beta}} d t^{\frac{n}{\beta}}\right)^{\frac{\beta}{n}} \leq\|f\|_{\Lambda_{\alpha, K}^{1,1}}, \quad \forall f \in C_{0}^{\infty} ;
$$

(ii) The geometric inequality

$$
\operatorname{cap}\left(\bar{O} ; \dot{\Lambda}_{\alpha, K}^{1,1}\right) \leq 2 P_{\alpha}(\bar{O}, K), \quad \text { for all bounded domain } O \subset \mathbb{R}^{n} \text { with } C^{\infty} \text { boundary } \partial O .
$$

Proof. We first prove that inequality (17) holds and is equivalent to inequality (16), and hence inequality (16) holds automatically.

The proof of inequality (17) is similar to that of Theorem 3.1 in [11]. For completeness, we include a brief proof here. Let $O \subset \mathbb{R}^{n}$ be a bounded domain with $C^{\infty}$ boundary $\partial O$. Recall that 
$\|\cdot\|$ is equivalent to $\|\cdot\|_{K}$ for any given origin-symmetric convex body $K$. By Lemma 3.2 in [11], for all $\epsilon>0$, one can find a function $g \in C_{0}^{\infty}$, such that, $0 \leq g \leq 1, g(x)=1$ for $x \in \bar{O}$ (which implies $g \geq \mathbf{1}_{\bar{O}}$ ), and

$$
\int_{O^{c}} \int_{O^{c}} \frac{|g(x)-g(y)|}{\|x-y\|_{K}^{n+\alpha}} d x d y<\epsilon .
$$

Hence, formulas (2) and (6), together with $g \in C_{0}^{\infty}$ and $g \geq \mathbf{1}_{\bar{O}}$, imply

$$
\begin{aligned}
\operatorname{cap}\left(\bar{O} ; \dot{\Lambda}_{\alpha, K}^{1,1}\right) & \leq \int_{\mathbb{R}^{n}} \int_{\mathbb{R}^{n}} \frac{|g(x)-g(y)|}{\|x-y\|_{K}^{n+\alpha}} d x d y \\
& \leq 2 \int_{O} \int_{O^{c}} \frac{|g(x)-g(y)|}{\|x-y\|_{K}^{n+\alpha}} d x d y+\int_{O^{c}} \int_{O^{c}} \frac{|g(x)-g(y)|}{\|x-y\|_{K}^{n+\alpha}} d x d y \\
& <2 P_{\alpha}(O, K)+\epsilon \\
& =2 P_{\alpha}(\bar{O}, K)+\epsilon .
\end{aligned}
$$

The desired inequality (177) follows by taking $\epsilon \rightarrow 0^{+}$.

Now we prove the equivalence between inequalities (16) and (17). First, we assume that inequality (16) holds true. Let $\epsilon \in(0,1)$ and $O \subset \mathbb{R}^{n}$ be a bounded domain with $C^{\infty}$ boundary $\partial O$. Let $O_{\epsilon}$ and $f_{\epsilon}$ be as in the proof of Theorem 6. Also note that $f_{\epsilon}(x)=1$ for all $x \in \bar{O}$, and hence $\bar{O} \subset \overline{O_{t}\left(f_{\epsilon}\right)}$ for all $\epsilon \in(0,1)$ and $t \in(0,1)$. By Part (ii) of Theorem 1 and inequality (16), one has

$$
\begin{aligned}
\operatorname{cap}\left(\bar{O} ; \dot{\Lambda}_{\alpha, K}^{1,1}\right) & \leq\left(\int_{0}^{1}\left(\operatorname{cap}\left(\overline{O_{t}\left(f_{\epsilon}\right)} ; \dot{\Lambda}_{\alpha, K}^{1,1}\right)\right)^{\frac{n}{\beta}} d t^{\frac{n}{\beta}}\right)^{\frac{\beta}{n}} \\
& \leq\left(\int_{0}^{\infty}\left(\operatorname{cap}\left(\overline{O_{t}\left(f_{\epsilon}\right)} ; \dot{\Lambda}_{\alpha, K}^{1,1}\right)\right)^{\frac{n}{\beta}} d t^{\frac{n}{\beta}}\right)^{\frac{\beta}{n}} \\
& \leq\left\|f_{\epsilon}\right\|_{\dot{\Lambda}_{\alpha, K}^{1,1}} .
\end{aligned}
$$

As $f_{\epsilon}(x) \rightarrow \mathbf{1}_{\bar{O}}$, the dominated convergent theorem implies the desired inequality (17):

$$
\operatorname{cap}\left(\bar{O} ; \dot{\Lambda}_{\alpha, K}^{1,1}\right) \leq \lim _{\epsilon \rightarrow 0^{+}}\left\|f_{\epsilon}\right\|_{\Lambda_{\alpha, K}^{1,1}}=\left\|\mathbf{1}_{\bar{O}}\right\|_{\dot{\Lambda}_{\alpha, K}^{1,1}}=2 P_{\alpha}(\bar{O}, K) .
$$

Second, we assume that inequality (17) holds. Note that $O_{t}(f) \subset O_{s}(f)$ holds for any function $f \in C_{0}^{\infty}$ and $0<s<t$. Part (ii) of Theorem 1 implies that $\operatorname{cap}\left(\overline{O_{t}(f)} ; \dot{\Lambda}_{\alpha, K}^{1,1}\right)$ is decreasing on $t \in[0, \infty)$. Hence,

$$
\begin{aligned}
t^{\frac{n}{\beta}-1}\left(\operatorname{cap}\left(\overline{O_{t}(f)} ; \dot{\Lambda}_{\alpha, K}^{1,1}\right)\right)^{\frac{n}{\beta}} & =\left(t \operatorname{cap}\left(\overline{O_{t}(f)} ; \dot{\Lambda}_{\alpha, K}^{1,1}\right)\right)^{\frac{n}{\beta}-1} \operatorname{cap}\left(\overline{O_{t}(f)} ; \dot{\Lambda}_{\alpha, K}^{1,1}\right) \\
& \leq\left(\int_{0}^{t} \operatorname{cap}\left(\overline{O_{s}(f)} ; \dot{\Lambda}_{\alpha, K}^{1,1}\right) d s\right)^{\frac{n}{\beta}-1} \operatorname{cap}\left(\overline{O_{t}(f)} ; \dot{\Lambda}_{\alpha, K}^{1,1}\right) \\
& =\frac{\beta}{n} \cdot \frac{d}{d t}\left(\int_{0}^{t} \operatorname{cap}\left(\overline{O_{s}(f)} ; \dot{\Lambda}_{\alpha, K}^{1,1}\right) d s\right)^{\frac{n}{\beta}} .
\end{aligned}
$$

Integrating the above inequality over $t \in(0, \infty)$, one has

$$
\int_{0}^{\infty}\left(\operatorname{cap}\left(\overline{O_{t}(f)} ; \dot{\Lambda}_{\alpha, K}^{1,1}\right)\right)^{\frac{n}{\beta}} d t^{\frac{n}{\beta}}=\frac{n}{\beta} \cdot \int_{0}^{\infty} t^{\frac{n}{\beta}-1}\left(\operatorname{cap}\left(\overline{O_{t}(f)} ; \dot{\Lambda}_{\alpha, K}^{1,1}\right)\right)^{\frac{n}{\beta}} d t
$$




$$
\begin{aligned}
& \leq \int_{0}^{\infty} \frac{d}{d t}\left(\int_{0}^{t} \operatorname{cap}\left(\overline{O_{s}(f)} ; \dot{\Lambda}_{\alpha, K}^{1,1}\right) d s\right)^{\frac{n}{\beta}} d t \\
& =\left(\int_{0}^{\infty} \operatorname{cap}\left(\overline{O_{s}(f)} ; \dot{\Lambda}_{\alpha, K}^{1,1}\right) d s\right)^{\frac{n}{\beta}} .
\end{aligned}
$$

Hence, inequality (17) and the co-area formula (10) imply the desired inequality (16):

$$
\begin{aligned}
\left(\int_{0}^{\infty}\left(\operatorname{cap}\left(\overline{O_{t}(f)} ; \dot{\Lambda}_{\alpha, K}^{1,1}\right)\right)^{\frac{n}{\beta}} d t^{\frac{n}{\beta}}\right)^{\frac{\beta}{n}} & \leq \int_{0}^{\infty} \operatorname{cap}\left(\overline{O_{t}(f)} ; \dot{\Lambda}_{\alpha, K}^{1,1}\right) d t \\
& \leq 2 \int_{0}^{\infty} P_{\alpha}\left(\overline{O_{t}(f)}, K\right) d t \\
& =2 \int_{0}^{\infty} P_{\alpha}\left(O_{t}(f), K\right) d t \\
& =\|f\|_{\dot{\Lambda}_{\alpha, K}^{1,1}} .
\end{aligned}
$$

Remark 6. Similar result for anisotropic Sobolev capacity cap $\left(\cdot, \dot{W}_{K}^{1,1}\right)$ also holds and is an extension of [20, Theorem 1.1]. More precisely, with $0<\beta<n$, the following inequalities hold and are equivalent:

(i) For all $f \in C_{0}^{\infty}$,

$$
\left(\int_{0}^{\infty}\left(\operatorname{cap}\left(\left\{x \in \mathbb{R}^{n}:|f(x)| \geq t\right\} ; \dot{W}_{K}^{1,1}\right)\right)^{\frac{n}{\beta}} d t^{\frac{n}{\beta}}\right)^{\frac{\beta}{n}} \leq \int_{\mathbb{R}^{n}}\|\nabla f(x)\|_{Z_{1}^{*} K} d x ;
$$

(ii) For all bounded domain $O \subset \mathbb{R}^{n}$ with $C^{\infty}$ boundary $\partial O$,

$$
\operatorname{cap}\left(\bar{O} ; \dot{W}_{K}^{1,1}\right) \leq 2 P\left(\bar{O}, Z_{1} K\right) .
$$

Finally, as a more general formulation of [22, Theorem 4] and [13, Theorem 9], we have the following equivalence.

Theorem 8. Let $\mu$ be a nonnegative Radon measure on $\mathbb{R}^{n}$, and $0<\beta \leq n$ and $\kappa_{n, \alpha, \beta}>0$ are constants. The following three inequalities are equivalent:

(i) The anisotropic fractional Sobolev inequality

$$
\|f\|_{L_{\mu}^{\frac{n}{\beta}}} \leq \kappa_{n, \alpha, \beta}\|f\|_{\Lambda_{\alpha, K}^{1,1}}, \text { for all } f \in C_{0}^{\infty} ;
$$

(ii) The anisotropic fractional isocapacitary inequality

$$
(\mu(\bar{O}))^{\frac{\beta}{n}} \leq \kappa_{n, \alpha, \beta} \operatorname{cap}\left(\bar{O}, \dot{\Lambda}_{\alpha, K}^{1,1}\right) \text {, for any bounded domain } O \subset \mathbb{R}^{n} \text { with } C^{\infty} \text { boundary } \partial O ;
$$

(iii) The anisotropic fractional isoperimetric inequality

$$
(\mu(\bar{O}))^{\frac{\beta}{n}} \leq 2 \kappa_{n, \alpha, \beta} P_{\alpha}(\bar{O}, K), \text { for any bounded domain } O \subset \mathbb{R}^{n} \text { with } C^{\infty} \text { boundary } \partial O .
$$

Proof. (i) $\Rightarrow$ (ii) Suppose that the anisotropic fractional Sobolev inequality in (i) holds true. Then, for all $f \in C_{0}^{\infty}$ with $f \geq \mathbf{1}_{\bar{O}}$, one has

$$
(\mu(\bar{O}))^{\frac{\beta}{n}}=\left(\int_{\mathbb{R}^{n}} \mathbf{1}_{\bar{O}} d \mu(x)\right)^{\frac{\beta}{n}} \leq\left(\int_{\mathbb{R}^{n}} f(x)^{\frac{n}{\beta}} d \mu(x)\right)^{\frac{\beta}{n}}=\|f\|_{L_{\mu}^{\frac{n}{\beta}}} \leq \kappa_{n, \alpha, \beta}\|f\|_{\Lambda_{\alpha, K}^{1,1}} .
$$


Taking the infimum over $f \in C_{0}^{\infty}$ with $f \geq \mathbf{1}_{\bar{O}}$ and by formula (2), one gets the desired anisotropic fractional isocapacitary inequality

$$
(\mu(\bar{O}))^{\frac{\beta}{n}} \leq \kappa_{n, \alpha, \beta} \operatorname{cap}\left(\bar{O}, \dot{\Lambda}_{\alpha, K}^{1,1}\right) .
$$

(ii) $\Rightarrow$ (iii) Assume that the anisotropic fractional isocapacitary inequality holds. Then, for any bounded domain $O \subset \mathbb{R}^{n}$ with $C^{\infty}$ boundary $\partial O$, one gets the desired anisotropic fractional isoperimetric inequality:

$$
(\mu(\bar{O}))^{\frac{\beta}{n}} \leq \kappa_{n, \alpha, \beta} \operatorname{cap}\left(\bar{O}, \dot{\Lambda}_{\alpha, K}^{1,1}\right) \leq 2 \kappa_{n, \alpha, \beta} P_{\alpha}(\bar{O}, K)
$$

where the last inequality follows from inequality (17).

(iii) $\Rightarrow$ (i) Assume that the anisotropic fractional isoperimetric inequality holds. Let $f \in C_{0}^{\infty}$ and $O_{t}(f)=\left\{x \in \mathbb{R}^{n}:|f(x)|>t\right\}$ for all $t \geq 0$. Obviously, $\mu\left(O_{t}(f)\right)$ is a decreasing function on $t \in[0, \infty)$, and hence for $0<\beta \leq n$,

$$
\left(\int_{0}^{t} \mu\left(O_{s}(f)\right) d s^{\frac{n}{\beta}}\right)^{\frac{\beta}{n}-1} \mu\left(O_{t}(f)\right) t^{\frac{n}{\beta}} \leq\left(\int_{0}^{t} \mu\left(O_{t}(f)\right) d s^{\frac{n}{\beta}}\right)^{\frac{\beta}{n}-1} \mu\left(O_{t}(f)\right) t^{\frac{n}{\beta}}=\left(\mu\left(O_{t}(f)\right)\right)^{\frac{\beta}{n}} t .
$$

Together with equality (14), one has

$$
\begin{aligned}
\|f\|_{L_{\mu}^{\frac{n}{\beta}}} & =\left(\int_{0}^{\infty} \mu\left(O_{t}(f)\right) d t^{\frac{n}{\beta}}\right)^{\frac{\beta}{n}} \\
& =\int_{0}^{\infty} \frac{d}{d t}\left(\int_{0}^{t} \mu\left(O_{s}(f)\right) d s^{\frac{n}{\beta}}\right)^{\frac{\beta}{n}} d t \\
& =\int_{0}^{\infty}\left(\int_{0}^{t} \mu\left(O_{s}(f)\right) d s^{\frac{n}{\beta}}\right)^{\frac{\beta}{n}-1} \mu\left(O_{t}(f)\right) t^{\frac{n}{\beta}-1} d t \\
& \leq \int_{0}^{\infty}\left(\mu\left(O_{t}(f)\right)\right)^{\frac{\beta}{n}} d t .
\end{aligned}
$$

Employing the anisotropic fractional isoperimetric inequality to $O_{t}(f)$, together with formulas (6) and (10), one gets, for all $f \in C_{0}^{\infty}$,

$$
\|f\|_{L_{\mu}^{\frac{n}{\beta}}} \leq \int_{0}^{\infty}\left(\mu\left(\overline{O_{t}(f)}\right)\right)^{\frac{\beta}{n}} d t \leq 2 \kappa_{n, \alpha, \beta} \int_{0}^{\infty} P_{\alpha}\left(O_{t}(f), K\right) d t=\kappa_{n, \alpha, \beta}\|f\|_{\dot{\Lambda}_{\alpha, K}^{1,1},},
$$

the desired anisotropic fractional Sobolev inequality.

Remark 7. Similarly, for a nonnegative Radon measure $\mu$, constants $0<\beta \leq n$ and $\kappa_{n, \beta}>0$, the following three inequalities are equivalent, whence extending [21, Proposition 3.1] (cf. [4, Propisition 3.1]):

(i) For all $f \in C_{0}^{\infty}$,

$$
\|f\|_{L_{\mu}^{\frac{n}{\beta}}} \leq \kappa_{n, \beta} \int_{\mathbb{R}^{n}}\|\nabla f(x)\|_{Z_{1}^{*} K} d x
$$

(ii) For any bounded domain $O \subset \mathbb{R}^{n}$ with $C^{\infty}$ boundary $\partial O$;

$$
(\mu(\bar{O}))^{\frac{\beta}{n}} \leq \kappa_{n, \beta} \operatorname{cap}\left(\bar{O}, \dot{W}_{K}^{1,1}\right) ;
$$

(iii) For any bounded domain $O \subset \mathbb{R}^{n}$ with $C^{\infty}$ boundary $\partial O$,

$$
(\mu(\bar{O}))^{\frac{\beta}{n}} \leq 2 \kappa_{n, \beta} P\left(\bar{O}, Z_{1} K\right)
$$




\section{REFERENCES}

[1] D. R. Adams, Lectures on $L^{p}$-potential Theory, Univ. of Umea, Dept. of Math. 2, 1981.

[2] D. R. Adams, Besov capacity redux, J. Math. Sci. (N.Y.) 162 (2009) 307-318.

[3] D. R. Adams and J. Xiao, Strong type estimates for homogeneous Besov capacities, Math. Ann. 325 (2003) 695-709.

[4] D. R. Adams and J. Xiao, The Hölder quasicontinuity for Riesz-Morrey potentials and Lane-Emden equations, arXiv: 1205.1987 .

[5] J. Bourgain, H. Brezis and P. Mironescu, Another look at Sobolev spaces, in Optimal Control and Partial Differential Equations (J. L. Menaldi, E. Rofman and A. Sulem, eds.), a volume in honour of A. Bensoussan's 60th birthday, IOS Press, 2001, pp. 439-455.

[6] J. Bourgain, H. Brezis and P. Mironescu, Limiting embedding theorems for $W^{s, p}$ when $s \uparrow 1$ and applications, J. Anal. Math. 87 (2002) 77-101.

[7] E. Di Nezza, G. Palatucci and E. Valdinoci, Hichhiker's guide to the fractional Sobolev spaces, Bull. Sci. math. 136 (2012) 521-573.

[8] A. Dinghas, Über einen geometrischen Satz von Wulff für die Gleichgewichtsform von Kristallen, Z. Kristallogr., Mineral. Petrogr. 105 (1944), no. Abt. A.

[9] A. Figalli, F. Maggi and A. Pratelli, A mass transportation approach to quantitative isoperimetric inequalities, Invent. Math. 182 (2010) 167-211.

[10] N. Fusco, V. Millot and M. Morini, A quantitative isoperimetric inequality for fractional perimeters, J. Funct. Anal. 261 (2011) 697-715.

[11] R. Hurri-Syrjänen and A.V. Vähäkangas, Characterizations to the fractional Sobolev inequality, arXiv: 1312.3135 .

[12] M. Ludwig, Anisotropic fractional Sobolev norms, Adv. Math. 252 (2014) 150-157.

[13] M. Ludwig, Anisotropic fractional perimeters, J. Differential Geom. 96 (2014) 77-93.

[14] V. Maz'ya, Sobolev Spaces with Applications to Elliptic Partial Differential Equations, 2nd, revised and augmented edition, Springer, 2011.

[15] V. Maz'ya and T. Shaposhnikova, On the Bourgain, Brezis, and Mironescu theorem concerning limiting embeddings of fractional Sobolev spaces, J. Funct. Anal. 195 (2002) 230-238.

[16] V. Maz'ya and T. Shaposhnikova, Erratum to: "On the Bourgain, Brezis, and Mironescu theorem concerning limiting embeddings of fractional Sobolev spaces" [J. Funct. Anal. 195 (2002) 230-238], J. Funct. Anal. 201 (2003) 298-300.

[17] P. Silvestre, Capacities and embeddings via symmetrization and conductor inequalities, Proc. Amer. Math. Soc. 142 (2014) 497-505.

[18] A. Visintin, Nonconvex functionals related to multiphase systems, SIAM J. Math. Anal. 21 (1990) 1281-1304.

[19] J. Xiao, Homogeneous endpoint Besov space embeddings by Hausdorff capacity and heat equation, Adv. Math. 207 (2006) 828-846.

[20] J. Xiao, The sharp Sobolev and isoperimetric inequalities split twice, Adv. Math. 211 (2007) 417-435 \& Corrigendum to "The sharp Sobolev and isoperimetric inequalities split twice" [Adv. Math. 211 (2007) 417-435] Adv. Math. (2014), http://dx.doi.or/10.1016/j.aim.2014.04.011

[21] J. Xiao, The $p$-Faber-Krahn inequality noted, In: Around the Research of Vladimir Maz'ya I. Function Spaces, pp. 373-390, Springer, 2010.

[22] J. Xiao, Optimal geometric estimates for fractional Sobolev capacities, arXiv: 1404.2245

Department of Mathematics and Statistics, Memorial University, St. John's, NL A1C 5S7, Canada

E-mail address: jxiao@mun.ca

Department of Mathematics and Statistics, Memorial University, St. John's, NL A1C 5S7, Canada

E-mail address: deping. ye@mun.ca 